\title{
LA HETEROGENEIDAD DEL PAISAJE DEL BORDE NORTE DE BOGOTÁ (COLOMBIA) AFECTA LA ACTIVIDAD DE LOS MURCIÉLAGOS INSECTÍVOROS
}

\section{LANDSCAPE HETEROGENEITY OF BOGOTÁ'S NORTHERN BORDER (COLOMBIA) AFFECTS INSECTIVOROUS BATS' ACTIVITY}

\author{
Francisco Sánchez ${ }^{1}$
}

${ }^{1}$ Ph.D. en Ciencias Ambientales. Facultad de Ciencias Ambientales, Universidad de Ciencias Aplicadas y Ambientales U.D.C.A, Calle 222 No. 55-37, Bogotá D.C., Colombia. frsanchez@udca.edu.co

Rev. U.D.C.A Act. E Div. Cient. 14(1): 71 - 80, 2011

\section{RESUMEN}

El borde norte de la Bogotá, Colombia, es un mosaico de ecosistemas transformados (urbanizaciones, cultivos, pastizales) y ecosistemas naturales (bosques andinos, humedales). En la actualidad, no se conoce cómo dicha heterogeneidad espacial afecta la ecología de las especies silvestres que sobreviven en la ciudad. Se propuso, que la heterogeneidad del paisaje del norte de Bogotá afecta el patrón temporal de actividad de los murciélagos insectívoros y su uso de hábitat. Se esperaba encontrar diferentes patrones de actividad temporal y de uso en sitios con diferentes grados de alteración humana. Se hicieron inspecciones acústicas en sitios con remanentes naturales, sitios con iluminación artificial y cercana a vías para automóviles. Los murciélagos insectívoros se registraron, tanto en áreas con remanentes naturales como intervenidas a lo largo del norte de Bogotá. La actividad de los murciélagos insectívoros cerca a vías importantes aumentó al avanzar la noche, mientras que en sitios sin vías principales fue en las primeras horas de la noche. Esto sugiere, que los murciélagos evitan zonas con alto flujo vehicular y estas vías reducen la disponibilidad de hábitats para los murciélagos insectívoros, al menos, en las primeras horas de la noche. Los sitios con iluminación artificial concentraron mayor actividad de forrajeo que sitios sin ella. La iluminación artificial parece beneficiar a algunos murciélagos, que encuentran allí parches, donde se acumulan sus presas potenciales y, es posible, que dicha iluminación se pueda usar en áreas agrícolas de la ciudad, para el control de poblaciones plaga, con la ayuda de los murciélagos.
Palabras clave: Andes, Chiroptera, ecolocalización, ecología urbana, seguimiento acústico.

\section{SUMMARY}

The northern border of Bogotá, Colombia, is a mosaic of transformed ecosystems (urbanizations, crops, pastures) and natural ecosystems (Andean forests, wetlands). Currently it is not known how such landscape heterogeneity affects the ecology of wild species that survive in the city. It was hypothesized that landscape heterogeneity affects insectivorous bats' activity and habitat use. It was expected to find different temporal activity patterns and use of sites with different degrees of human intervention. To test these predictions, acoustic monitoring was used at natural ecosystems, sites with artificial illumination and near highways. Insectivorous bats were found both in natural and transformed ecosystems throughout Bogotá's northern border. Insectivorous bats' activity near highways increased toward midnight, whereas in areas with no major streets activity was higher few hours after sunset. This suggests that bats avoid areas with high vehicular flow and they reduce habitat availability for bats, at least during the first hours of the night. Sites with artificial illumination had higher foraging activity than places without it. Thus, the presence of artificial illumination seems to benefit some bats that encounter patches where potential prey accumulate, and it is possible that such illumination may be used in agricultural areas of the city to control pests with the bats' help. 
Key words: Andes, Chiroptera, echolocation, urban ecology, acoustic monitoring.

\section{INTRODUCCIÓN}

Bogotá tiene más de siete millones de habitantes, es una de las ciudades más grandes de Latinoamérica y el crecimiento de su población humana ha causado la reducción en el área de sus ecosistemas naturales (Mendosa E Etter, 2002). Por ejemplo, el paisaje del borde norte de Bogotá, se caracteriza por ser un mosaico de ecosistemas transformados y naturales y entre los transformados, se cuentan áreas urbanizadas y áreas dedicadas a la agricultura y/o la ganadería (Ramírez et al. 2008). Aunque gran parte de los hábitats naturales han desaparecido de la ciudad, en el borde norte de Bogotá aun se conservan remanentes importantes de ambientes de montaña, como los Cerros Orientales y el Cerro de la Conejera y algunos humedales, como Juan Amarillo, La Conejera y el complejo Torca-Guaymaral; existe un remanente de bosque de planicie flaviolacustre en la Hacienda Las Mercedes (Ramírez et al. 2008; Secretaría Distrital de Ambiente, 2007). Dichos ecosistemas son reservorios de recursos naturales y biodiversidad, reconocidos como estratégicos para Bogotá y sus habitantes, en el plan de ordenamiento de la ciudad (Alcaldía Mayor de Bogotá, Decreto 619 de 2000). A pesar de su importancia, es poco lo que se conoce sobre la ecología e historia natural de las especies silvestres que aún sobreviven en los ecosistemas naturales mencionados o en Bogotá, en general, con excepción, tal vez, de las aves (ABO, 2000). Dicha falta de información crea serias dificultades a la hora de proponer planes de manejo o de conservación de las especies silvestres de cualquier área (Pullin, 2002).

Por ejemplo, no se conoce cómo el mosaico de ecosistemas del norte de Bogotá afecta la ecología de los animales silvestres que allí se viven. Un caso particular son los murciélagos, que por sus hábitos nocturnos y por su baja abundancia en comparación con zonas cálidas, pasan desapercibidos para muchos de los habitantes de Bogotá (autor, datos sin publicar); sin embargo, para Bogotá, hay reportadas once especies: cuatro de la familia Vespertilionidae (Eptesicus fuscus, Myotis nigricans, Lasiurus cinereus, Histiotus montanus), tres de la familia Molossidae (Eumops glaucinus, Nyctinomops aurispinosus, Tadarida brasiliensis) y cuatro de la familia Phyllostomidae; Anoura geoffroyi, Carollia perspicillata, Sturnira ludovici, S. bogotensis (Pacheco E Patterson, 1992; Tamsitt E Valdivieso, 1963; Tamsitt et al. 1964). Las especies de las familias Vespertilionidae y Molossidae son estrictamente insectívoras y todas cazan sus presas al vuelo (insectívoros aéreos), con excepción de $H$. montanus, que las atrapa iniciando el vuelo desde perchas y capturándolas sobre superficies (animalívoro del follaje)
(Norberg \& Rayner, 1987). Por su parte, las especies de la familia Phyllostomidae, registradas para Bogotá, pueden consumir frutas, néctar y polen y complementan su dieta con insectos (Gardner, 1977).

El estudio de la ecología de los murciélagos puede traer múltiples beneficios para aquellos interesados en el manejo y la conservación de un área. Los murciélagos insectívoros pueden prestar servicios ambientales relacionados con su papel como depredadores de algunas especies, que pueden ser consideradas plagas agrícolas o transmisoras de enfermedades contagiosas (Cleveland et al. 2006; Tuttle $\varepsilon$ Moreno, 2007), mientras que los herbívoros pueden ser dispersores de semillas y/o polinizadores (Fleming, 1993). Adicionalmente, los murciélagos han sido usados como indicadores de la calidad de los hábitats. La composición y la abundancia de los murciélagos se han relacionado con diferentes tipos de disturbio humano, como por ejemplo, la intensificación de la degradación de hábitats naturales (Fenton et al. 1992; Medellín et al. 2000) o el uso de diferentes tipos de agricultura (Jennings \& Pocock, 2009). De igual manera, se ha encontrado que el comportamiento de los murciélagos también puede ser afectado por la actividad antrópica y el desarrollo urbano.

En ambientes fragmentados de México, los murciélagos insectívoros tienen mayores niveles de actividad en los asentamientos humanos, fragmentos de selva y cercas vivas que en zonas con pastizales (Estrada et al. 2004). Por otra parte, el efecto del desarrollo urbano en áreas cercanas a remanentes de bosques sobre los murciélagos insectívoros parece depender de la especie (Jung $\&$ Kalko, 2010). Así, algunos murciélagos, parecen tolerar las urbanizaciones y aprovechan la presencia de luces artificiales, que atraen insectos, como sitios de forrajeo, aunque la rentabilidad parece ser mejor alrededor de las lámparas de luz blanca, que alrededor de las de luz amarilla-naranja (Jung \& Kalko, 2010; Rydell, 2006); sin embargo, los murciélagos también pueden ser perjudicados por las áreas urbanizadas, que vienen asociadas con la construcción de vías y éstas pueden ser agentes de mortalidad para los murciélagos insectívoros que sufren el riesgo de estrellarse con los vehículos (Lodé, 2000; Russell et al. 2009). Por lo tanto, el desarrollo urbanístico puede, potencialmente, tener efectos positivos y negativos sobre las poblaciones de murciélagos insectívoros.

Con base en lo anterior, se propone que la heterogeneidad del paisaje del borde norte de Bogotá afecta el patrón temporal de actividad de los murciélagos insectívoros y su uso de hábitat. Esto es porque existen sitios cerca de vías que podrían ser percibidos por los murciélagos, como áreas con alto de riesgo de depredación o muerte (Adams et al. 2006; Zurcher et al. 2010) y, probablemente, sean más 
usados al reducirse el flujo vehicular. Además, los sitios con iluminación artificial pueden beneficiar el forrajeo de los murciélagos insectívoros, por lo que se espera que sean más usados en actividades de alimentación que sitios sin iluminación artificial.

\section{MATERIALES Y MÉTODOS}

Sitios de muestreo: Para evaluar las predicciones, se muestrearon cinco sitios con diferentes grados de alteración antrópica.

- Campus norte de la Universidad de Ciencias Aplicadas y Ambientales U.D.C.A (2.554 m.s.n.m.). Los muestreos, se realizaron en septiembre-noviembre de 2008, por diez noches, una vez a la semana, cerca a un edificio con lámparas de luz blanca, i.e., de vapor de mercurio. El campus está entre la zona de expansión urbana y rural de Bogotá y cuenta con campos de fútbol y algunas edificaciones. Alrededor del campus, se viene formando, desde hace aproximadamente dos años, una cerca viva, alrededor de $2 \mathrm{~m}$ de ancho, con varias especies de plantas nativas. El campus está cerca del Humedal TorcaGuaymaral, 1-1,5 km.

- Separador de la Autopista Norte, entre calles 220 y 226 (2.560 m.s.n.m.). Los muestreos fueron en febrero de 2010, una o dos veces a la semana hasta completar cinco noches, simultáneamente, en un área con un remanente de humedal y un bosque de árboles plantados (programa Hojas Verdes). El separador está entre vías de tres carriles en sentido sur-norte y tres en sentido norte-sur, con lámparas de luz naranja (i.e., de vapor de sodio), a lo largo de ambos lados de la vía. La zona plantada tiene árboles de 5-12m, mientras que la zona de humedal tiene vegetación hidrofítica típica, e.g., Cyperaceae y Juncaceae (Osbahr \& Hernández Schmidt, 2006). En la zona de humedal existe una franja divisoria en sentido norte-sur de Penisetum clandestinum, pasto dominante del estrato herbáceo en la zona.

- Base del Cerro de Torca ( Carrera $7^{\circ}$ con calle 235 ( $\sim 2.650$ m.s.n.m). Se realizaron tres muestreos, una vez a la semana, en marzo de 2010, simultáneamente, cerca a una lámpara de luz naranja sobre la carrera séptima y cerca al borde de un bosque andino secundario. Junto al bosque andino, se presentan plantaciones de árboles exóticos y áreas de potreros, con algunas casas. La disposición de la Carrera $7^{\mathrm{a}}$, una vía con un carril sur-norte y uno norte-sur, genera una discontinuidad marcada entre la vegetación al oriente y occidente de esta vía. A lo largo de la vía hay lámparas de luz naranja.
- Hacienda Las Mercedes-Humedal La Conejera ( 2.600 m.s.n.m). Los muestreos fueron en marzo-abril de 2010, una vez a la semana hasta completar cinco noches, en un remanente de bosque bajo, secundario de planicie flaviolacustre y cerca a una casa, con un humedal artificial y una lámpara de luz blanca; ambos sitios están rodeados de pastizales, zonas de cultivo y plantaciones de árboles exóticos. El bosque de planicie flaviolacustre está dominado por árboles de 8-15m; el estrato herbáceo tiene una buena representación de $P$. clandestinum, mientras que el estrato arbustivo tiene sectores abundantes en enredaderas. También se muestreó en el límite de Las Mercedes con el Humedal La Conejera, una vez a la semana, hasta completar cinco noches, simultáneamente, en un borde humedalpastizal y a la orilla del humedal. En el área de límite entre Las Mercedes y el Humedal concurren jarillones, que evitan la inundación de las tierras de la Hacienda y sobre éstos, árboles plantados y dispersos. En el Humedal se presenta vegetación hidrofítica típica, e.g., Cyperaceae y Juncaceae.

- Floresta de la Sabana-Cerros Orientales (oriente de la Carrera $7^{a}$, límite con el municipio de La Calera $(\sim 2.800$ m.s.n.m.). Los muestreos fueron en mayo-junio de 2010, una vez a la semana, hasta completar tres noches, simultáneamente, en el borde de un bosque altoandino y cerca a una lámpara de luz naranja sobre una carretera. El bosque altoandino es parte de la Reserva Forestal de los Cerros Orientales (CAR, 2006). En el bosque subsisten árboles de 10-20m, con un dosel discontinuo y con alta acumulación de materia orgánica sobre el suelo. Las epífitas vasculares y no vasculares son abundantes, al igual que los líquenes. Al borde de los caminos, se puede observar retamo espinoso Ulex europaeus, en algunos sectores. Dividiendo el bosque, se encuentran vías de dos carriles, de $<6 \mathrm{~m}$ de ancho y con lámparas de luz naranja a su largo.

Estudio de los murciélagos: Se hicieron puntos de observación de 10 minutos (Avila-Flores E Fenton, 2005), en los que se usó un detector de murciélagos Batbox duet (Batbox Ltd., West Sussex, England), para registrar las llamadas ultrasónicas. En la U.D.C.A, se hicieron estaciones de cinco minutos. Los puntos de observación fueron cada hora, desde las 18h30min hasta las 24h30min, con excepción de la U.D.C.A, donde iniciaron a las 19h30min. En horas con alta precipitación, se detuvo el muestreo.

Los murciélagos insectívoros recurren a llamadas de alta frecuencia para ubicarse en el espacio y para localizar y capturar a sus presas (Fenton, 1974). La ecolocalización en los murciélagos insectívoros, se puede dividir en tres fases (Altringham, 2001): la primera es la de búsqueda, en la que el murciélago rastrea a presas o se desplaza; en esta fase, la tasa de emisión de llamadas (pulsos) y las frecuencias 
empleadas, generalmente, varían poco; la segunda es la fase de enfoque, que inicia después de detectar una presa; la tasa de repetición de los pulsos aumenta y la duración de cada pulso e interpulso, decrece en esta fase y, en la terminal, las variables mencionadas llegan a valores extremos, antes de tratar de capturar la presa.

Con el detector de murciélagos, se pueden distinguir fases de búsqueda de las terminales por el cambio en la tasa de emisión de pulsos; así, se reconocen comportamientos asociados al desplazamiento o búsqueda de alimento (fases de búsqueda) y comportamientos asociados a intentos de captura o de forrajeo (fases terminales). Por ello, en cada estación de observación, se registró el número de fases de búsqueda y de fases terminales. Durante cada estación, se varió la frecuencia reconocida por el detector, $17-120 \mathrm{kHz}$, mientras el micrófono de detector, se apuntaba hacia el cielo y perpendicular al suelo.

Análisis estadísticos: Para analizar los patrones temporales de actividad, se aplicaron análisis de varianza independientes por sitio. En cada análisis, la variable dependiente era el número de fases de búsqueda o de fases terminales, por cinco o diez minutos. Cuando los supuestos de normalidad y/o homogeneidad de varianza no se cumplieron, se elaboraron transformaciones logarítmicas de las variables dependientes (Zar, 1999). Se incluyó la hora, como factor fijo y el punto de observación, como aleatorio, dado que se tomaban varias medidas en cada punto, mientras que la noche de muestreo entró como una variable bloque. Se incluyó la iluminación de la luna por noche (Time and Date AS, en línea), como co-variable, pero en ninguno de los análisis, se evidenció un efecto significativo y, en aquellos casos en que su remoción no afectaba, considerablemente, los cuadrados medios de las otras variables independientes, este efecto fue removido del modelo. Con la misma lógica, la interacción entre hora y lugar fue incluida o no en el modelo estadístico.

Para complementar los análisis, se examinó la variación espacial del forrajeo de los murciélagos, con una prueba de Kruskal-Wallis (Zar, 1999), con el número de fases terminales/10min, a la hora de mayor actividad, como variable dependiente y, el punto de observación, como independiente. Seguido al análisis, se aplicaron pruebas a posteriori no paramétricas. La hora con mayor actividad, se definió con los análisis explicados en la sección anterior. El nivel de significancia en todas las pruebas estadísticas fue 0,05.

\section{RESULTADOS Y DISCUSIÓN}

Murciélagos insectívoros en el norte de Bogotá: Se registraron murciélagos insectívoros en todos los sitios; generalmente, se escucharon a frecuencias inferiores a los $45 \mathrm{kHz}$ y tenían su frecuencia con mayor energía alrededor de los $20 \mathrm{kHz}$; sólo en la Floresta de la Sabana, se escuchó un murciélago con la frecuencia de mayor energía, alrededor de $30 \mathrm{kHz}$ y un ancho de banda mucho más amplio que los otros murciélagos. Los resultados sugieren que existe, al menos, una especie de murciélago ampliamente distribuido y dadas las especies reportadas para Bogotá (Tamsitt et al. 1964) y las características de las llamadas, es posible que la o las especies que ecolocalizan alrededor de los $20 \mathrm{kHz}$ sea(n) de la familia Molossidae, ya que, por lo general, las especies de esta familia usan llamadas de baja frecuencia (Fenton $\mathcal{E}$ Bell, 1981). Por su parte, la especie que ecolocaliza alrededor de los $30 \mathrm{kHz}$ y parece tener un mayor ancho de banda es probablemente de la familia Vespertilionidae. Es necesario capturar dichos murciélagos y grabar sus llamadas en vuelo para evaluar estas afirmaciones.

Actividad de los murciélagos: En el separador de la Autopista, el punto de observación (humedal o bosque plantado) no tuvo efecto sobre la actividad de búsqueda o de forrajeo $\left(\mathrm{F}_{1}\right.$ ${ }_{6}=2,84, \mathrm{p}=0,14$ y $\mathrm{F}_{1,6}=0,01, \mathrm{p}=0,93$, respectivamente). La actividad de búsqueda y de forrajeo sí fue afectada por la hora $\left(\mathrm{F}_{6,7}=7,18, \mathrm{p}=0,01\right.$ y $\mathrm{F}_{6,7}=7,41, \mathrm{p}<0,01$, respectivamente) $\mathrm{y}$, en ambos casos, hubo una tendencia a aumentar la actividad, con el paso del tiempo (Figura 1A, B). En la Autopista, la noche tuvo un efecto significativo sobre la actividad de búsqueda y de forrajeo $\left(\mathrm{F}_{4,47}=5,59, \mathrm{p}<0,01 \mathrm{y}\right.$ $\mathrm{F}_{4,47}=4,42, \mathrm{p}<0,01$, respectivamente); en Torca, la actividad de búsqueda fue afectada por la interacción entre hora y punto de observación $\left(\mathrm{F}_{6,25}=3,81, \mathrm{p}<0,01\right)$, dado que no se detectaron cambios en la actividad al borde del bosque andino, mientras que cerca a la lámpara, la actividad fue significativamente mayor, a las 00h30min que en el resto de horas, cerca de la lámpara o en el bosque (Figura 1C). Los factores hora, punto de observación y noche no tuvieron efecto sobre la actividad de búsqueda (todos los p>0,05). Por su parte, la actividad de forrajeo sí fue afectada por la hora $\left(F_{6,31}=2,97, p=0,02\right)$ y el punto de observación $\left(F_{1}\right.$ $\left.{ }_{31}=9,183, p=0,05\right)$, mientras que la noche no tuvo efecto $\left(\mathrm{F}_{2,31}=1,39, \mathrm{p}=0,27\right)$. En Torca, la actividad de forrajeo fue mayor al lado de la lámpara sobre la Carrera $7^{\mathrm{a}}$ que al borde de bosque y significativamente mayor, a las 00h30min que a las 19h30min y 20h30min (Figura 1D). En Las MercedesHumedal La Conejera, la actividad de búsqueda fue afectada por la hora $\left(\mathrm{F}_{6,107}=3,07, \mathrm{p}<0,01\right)$ y por el punto de observación $\left(\mathrm{F}_{2,107}=6,35, \mathrm{p}<0,01\right)$, mientras que la noche no tuvo efecto $\left(\mathrm{F}_{8,107}=1,28, \mathrm{p}=0,61\right)$. La actividad de búsqueda fue mayor a las $18 \mathrm{~h} 30 \mathrm{~min}$ que a las 19h30min, 21h30min, 22h30min y 00h30min y fue mayor cerca a la lámpara de luz blanca que en el bosque, en el borde del humedal o a la orilla del humedal (Figura 2A). Por su parte, la actividad de forrajeo fue afectada por el punto de observación $\left(F_{2,108}=3,76\right.$, 
$\mathrm{p}=0,03)$ y no fue afectada significativamente por la hora o la noche $\left(\mathrm{F}_{6,108}=1,10, \mathrm{p}=0,37\right.$ y $\left.\mathrm{F}_{8,108}=1,46, \mathrm{p}=0,18\right)$. La actividad de forrajeo fue significativamente mayor, cerca de la lámpara de luz blanca respecto a los otros lugares de Las Mercedes (Figura 2B). En La Floresta, la actividad de búsqueda fue afectada por la hora $\left(\mathrm{F}_{6,26}=4,13, \mathrm{p}<0,01\right)$ y el punto de observación $\left(\mathrm{F}_{1,26}=5,93, \mathrm{p}=0,02\right)$, mientras que la noche no tuvo efecto $\left(\mathrm{F}_{2,26}=1,06, \mathrm{p}=0,36\right)$. La actividad de búsqueda fue mayor a las $18 \mathrm{~h} 30 \mathrm{~min}$ que a las $22 \mathrm{~h} 30 \mathrm{~min}$, 23h30min y 00h30min y fue mayor al borde del bosque andino que cerca a la lámpara de luz naranja (Figura $2 \mathrm{C}$ ). La actividad de forrajeo fue afectada por la hora $\left(\mathrm{F}_{6,26}=\right.$ $2,61, p=0,04)$, mientras que el punto de observación y la noche no tuvieron efecto $\left(F_{1,26}=1,77, p=0,19\right.$ y $F_{2,26}=0,79$, $p=0,46$, respectivamente). En la Floresta, la actividad de forrajeo fue mayor a las $18 \mathrm{~h} 30 \mathrm{~min}$ que a las $20 \mathrm{~h} 30 \mathrm{~min}$ y 21h30min (Figura 2D). En la U.D.C.A, la actividad de forrajeo fue afectada por la noche $\left(\mathrm{F}_{9,50}=2,71, \mathrm{p}=0,01\right)$ y por la hora $\left(\mathrm{F}_{5,50}=5,15, \mathrm{p}<0,01\right)$ y fue mayor a las $19 \mathrm{~h} 30 \mathrm{~min}$ que a las otras horas, con excepción de las 20h30min (Figuras 3A).
El análisis adicional para examinar la variación de la actividad de forrajeo entre los puntos de observación, a la hora de mayor actividad, muestra que ésta fue diferente entre los puntos ( $\mathrm{H}=29,7, \mathrm{p}<0,01)$ (Figura 3B). En la U.D.C.A hubo mayor actividad que en los otros puntos $(p<0,05)$, excepto los sitios con lámparas en Las Mercedes y en Torca. Cerca a la lámpara en Torca, se registró significativamente mayor actividad que a la orilla del Humedal La Conejera y el sitio con la lámpara en La Floresta $(p<0,05)$ y, estuvo cerca de ser significativamente mayor que al borde del humedal, el bosque de La Floresta y el bosque en Torca $(0,05>p<0,09)$. Cerca a la lámpara de Las Mercedes, la actividad estuvo cerca de ser significativamente mayor que a la orilla del Humedal, el bosque en Torca y el sitio con la lámpara en La Floresta $(0,06>p<1,0)$. Estos resultados, junto con los presentados en la sección anterior, sugieren que los sitios con lámparas de luz son aprovechados, considerablemente, por los murciélagos, para obtener alimento, pero su uso depende de la presencia de vías, como en el caso del separador de la autopista y la Carrera $7^{\mathrm{a}}$ y, posiblemente, de la altitud, como en el caso de La Floresta.
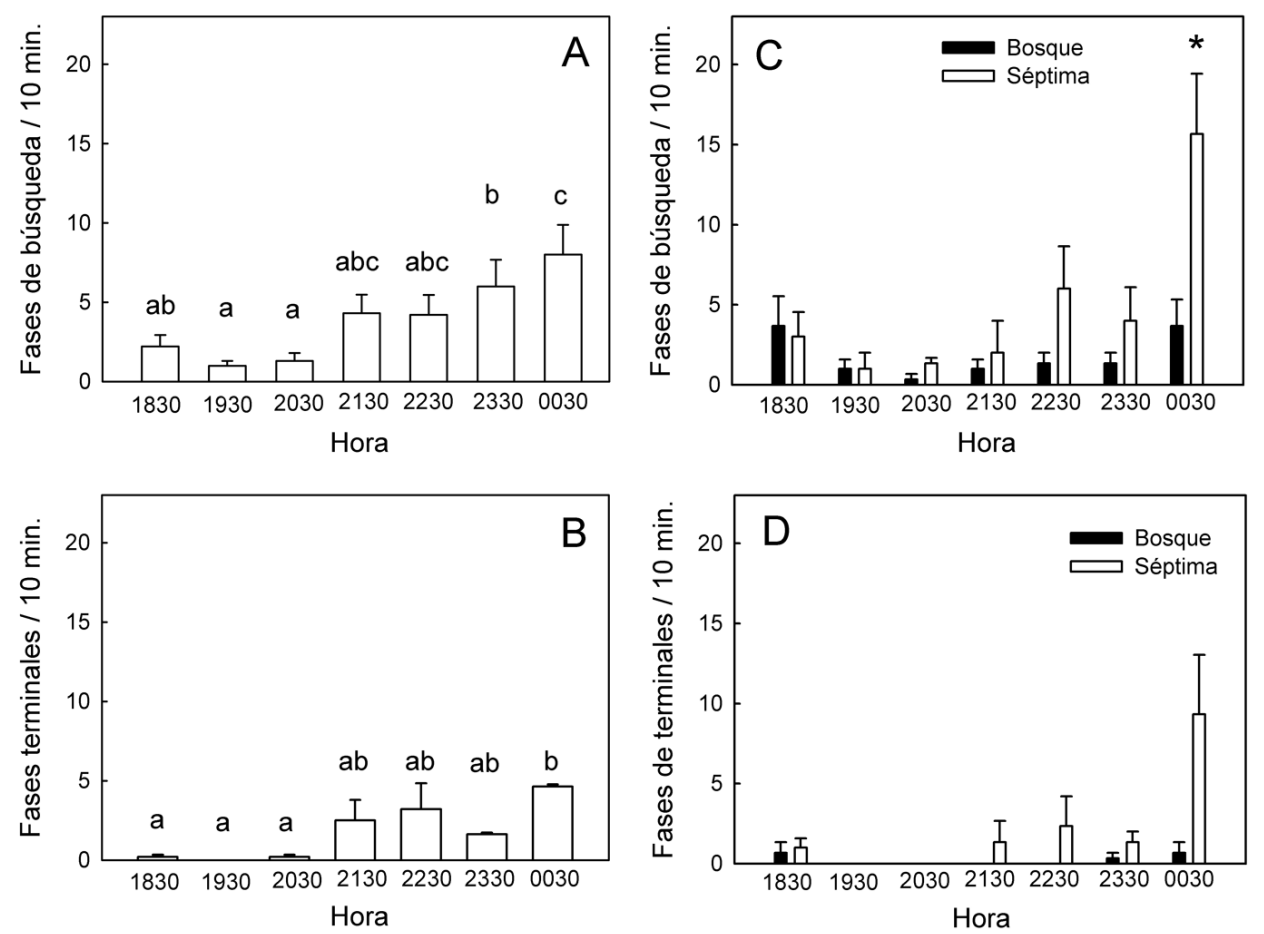

Figura 1. Actividad de los murciélagos insectívoros en el separador de la Autopista $(A, B)$ y en Torca $(C, D)$. La actividad expresada en número de fases de búsqueda (actividad de búsqueda) o fases terminales (actividad de forrajeo) en $10 \mathrm{~min}$. Las barras representan promedios \pm error estándar y las barras en $\mathrm{A}$ y $\mathrm{B}$, con letras diferentes, indican diferencias significativas (Prueba de Bonferroni, $p<0,05$ ). En la Autopista no hubo diferencias entre sitios, pero sí entre horas, con una tendencia a aumentar la actividad de búsqueda y forrajeo al progresar la noche (A, B). En Torca, la interacción entre sitio y hora fue significativa; la actividad de búsqueda fue mayor a las $00 \mathrm{~h} 30 \mathrm{~min}$ en la carrera. $7^{\mathrm{a}}$ que en el resto de las horas y sitios (C). La actividad de forrajeo fue mayor sobre la $7^{\mathrm{a}}$ que en el borde de bosque y mayor a las $00 \mathrm{~h} 30 \mathrm{~min}$ que a las $19 \mathrm{~h} 30 \mathrm{~min}$ y $20 \mathrm{~h} 30 \mathrm{~min}$. 

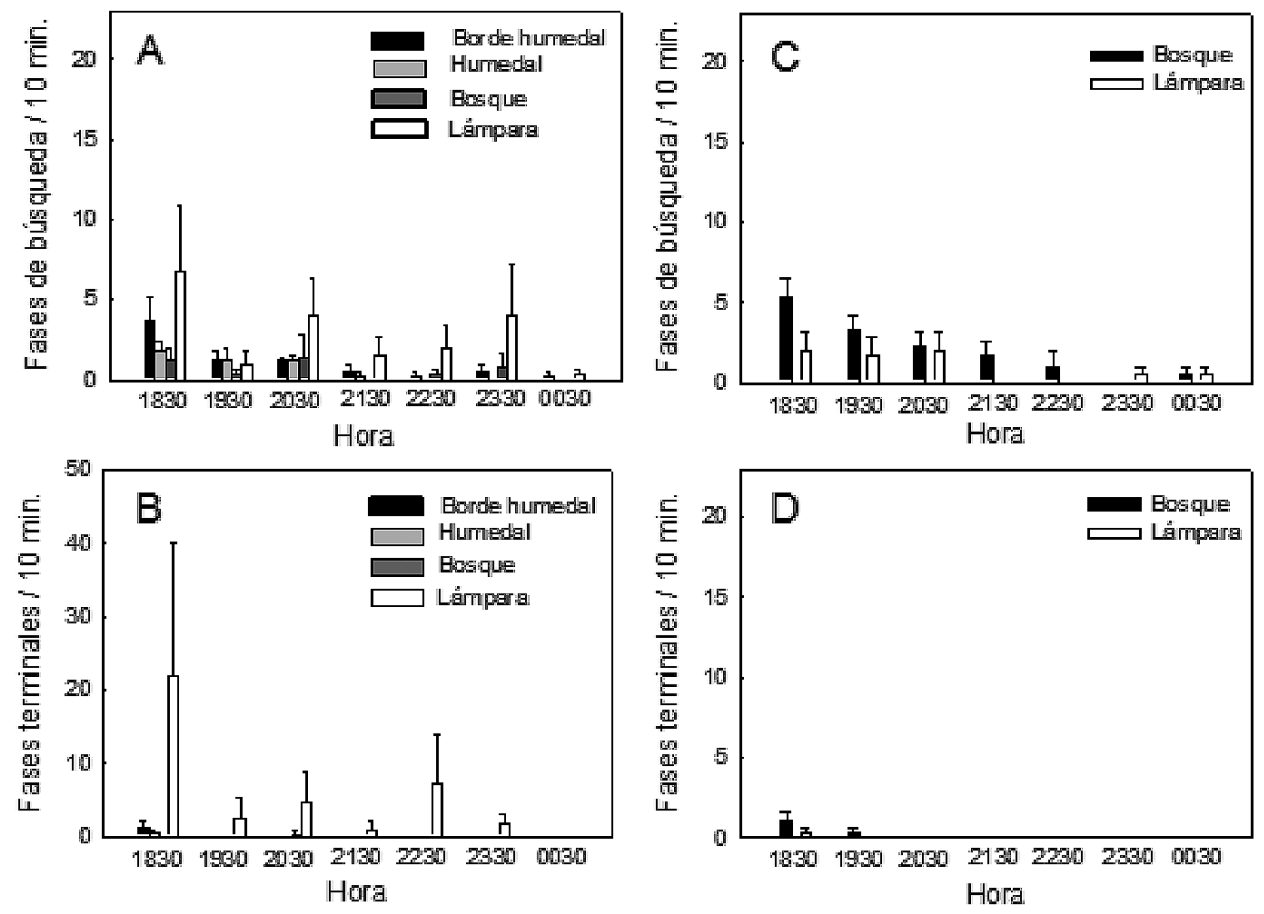

Figura 2. Actividad de los murciélagos insectívoros en La Hacienda Las Mercedes (A, B) y en la Floresta de La Sabana (C, D). La actividad expresada en número de fases de búsqueda (actividad de búsqueda) o fases terminales (actividad de forrajeo) en 10 min. Las barras representan promedios \pm error estándar. En Las Mercedes, la actividad de búsqueda y forrajeo fue mayor (Prueba de Bonferroni, $p<0,05$ ), cerca de una casa, con una lámpara de luz blanca que en el borde humedal-pastizal, a la orilla del humedal o en un remanente de bosque secundario (A, B). En Las Mercedes, la actividad de búsqueda fue mayor a las 18h30min que a las $19 \mathrm{~h} 30 \mathrm{~min}, 21 \mathrm{~h} 30 \mathrm{~min}, 22 \mathrm{~h} 30 \mathrm{~min}$ y $24 \mathrm{~h} 30 \mathrm{~min}$; la hora no afectó la actividad de forrajeo. En la Floresta hubo mayor actividad de búsqueda al borde de bosque que cerca a una lámpara de luz naranja; el sitio no afectó la actividad de forrajeo (C, D). En La Floresta, la actividad de búsqueda fue mayor a las 18h30min, que a las $22 \mathrm{~h} 30 \mathrm{~min}, 23 \mathrm{~h} 30 \mathrm{~min}$ y $24 \mathrm{~h} 30 \mathrm{~min}$, mientras que la actividad de forrajeo fue mayor a las $18 \mathrm{~h} 30 \mathrm{~min}$ que a las $20 \mathrm{~h} 30 \mathrm{~min}$ y $21 \mathrm{~h} 30 \mathrm{~min}$.

Aunque son necesarios estudios adicionales, dado que los muestreos fueron realizados en diferentes momentos, lo que podría implicar un posible efecto temporal y se emplearon relativamente pocos días por sitio, esta primera aproximación permite vislumbrar algunos posibles efectos de la heterogeneidad del paisaje bogotano, sobre la actividad de los murciélagos. Como se esperaba, los murciélagos tuvieron patrones temporales de actividad en el separador de la Autopista Norte y en la carrera $7^{\mathrm{a}}$, diferentes a los de los otros sitios. En la Autopista y la $7^{\text {a }}$, la actividad de los murciélagos aumentó al avanzar la noche, i.e., cuando el flujo vehicular decrecía, mientras que en los otros sitios, la actividad fue mayor en las horas siguientes al atardecer. El patrón temporal encontrado en la Autopista y en la $7^{\text {a }}$ contrasta, considerablemente, con lo observado en murciélagos insectívoros, en lo usual. En estos murciélagos es común encontrar un pico de actividad en las primeras horas de la noche, i.e., como en los sitios sin vías de alto flujo vehicular. Esto es debido a que animales con altos requerimientos energéticos, asociados a su pequeño tamaño y al vuelo, tienen una gran necesidad de alimentarse, luego de un día sin comida y porque a esas horas está la mayor actividad de insectos (Brown, 1968; Erkert, 1978; Kunz, 1973). Así, el patrón de actividad observado en la Autopista y en la carrera $7^{a}$ sugiere que los murciélagos perciben una amenaza en estos sitios y evitan las horas en las que habría un mayor riesgo de colisión con vehículos (Russell et al. 2009). En un estudio realizado en Norte América, Zurcher et al. (2010) percibieron que los murciélagos insectívoros muestran comportamientos evasivos hacia vías con alto tráfico vehicular estando o no en peligro inminente de colisión. Los mismos autores mencionan que, por lo anterior, las vías con alto tráfico vehicular se pueden convertir en barreras para los movimientos de los murciélagos. Así, es posible que en el norte de Bogotá, vías como la Autopista y la $7^{\mathrm{a}}$ sean barreras para su movimiento, al menos, durante ciertas horas de la noche.

Los resultados también muestran que los murciélagos insectívoros pueden detectar diferencias entre lugares 

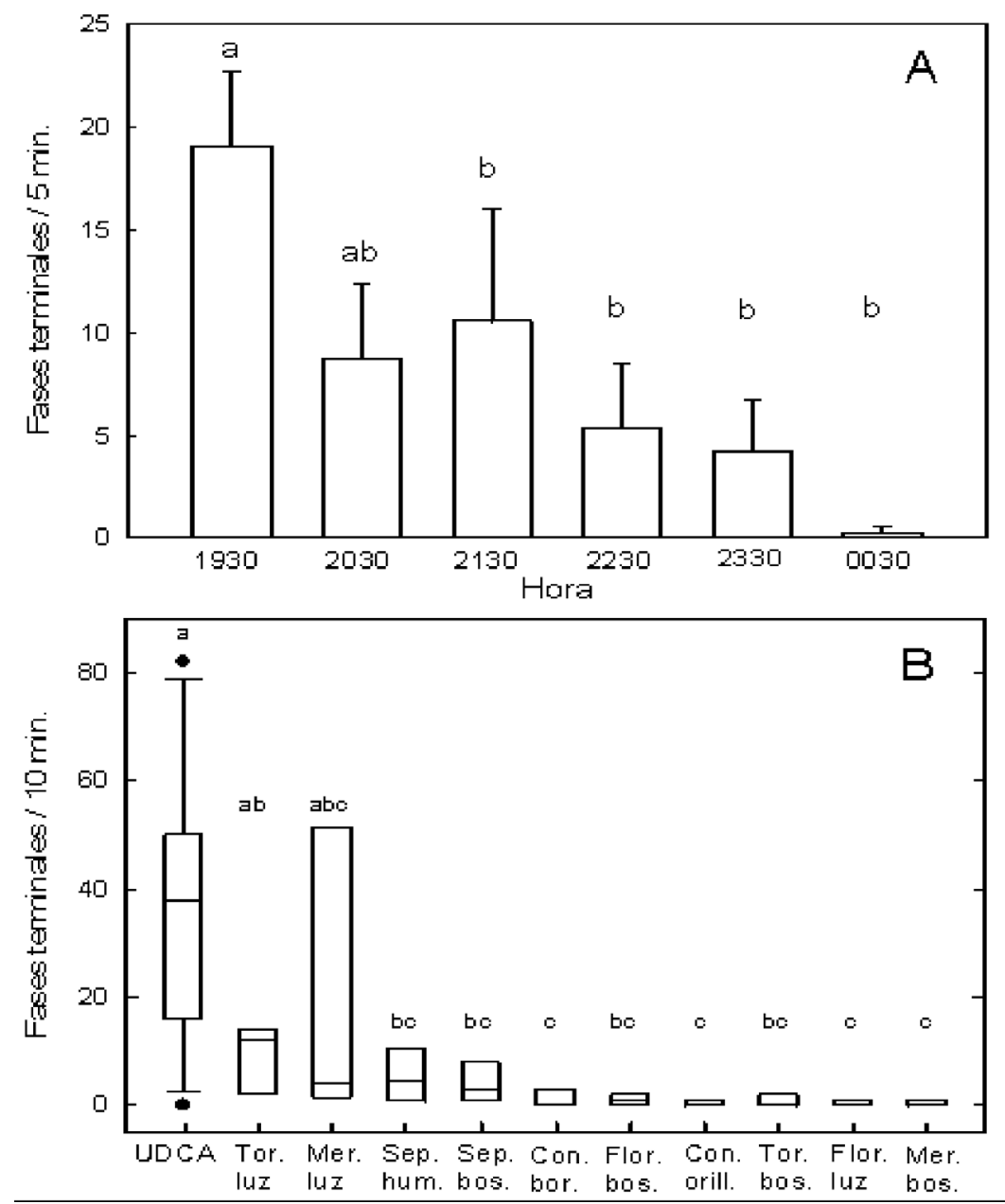

Figura 3. A. Actividad de forrajeo de los murciélagos insectívoros (fases de terminales/5 min), alrededor de un edificio con lámparas de luz blanca en la U.D.C.A. Las barras son promedios \pm error estándar; barras con letras diferentes indican diferencias significativas (Prueba de Bonferroni, $p<0,05$ ). La actividad tiende a disminuir con el tiempo. B. Actividad de forrajeo a la hora de mayor actividad en U.D.C.A, lámpara de luz en Torca (Carrera $7^{\mathrm{a}}$ ), Las Mercedes con lámpara de luz, humedal en el separador de la Autopista, bosque del separador, borde del humedal La Conejera, bosque en la Floresta, orilla humedal La Conejera, borde de bosque en Torca, lámpara de luz en la Floresta y bosque de Las Mercedes. La línea dentro de la caja es la mediana, sus extremos son los percentiles 25 y 75 , la barra de error representa los percentiles 10 y 90 y los puntos son valores atípicos. Las letras diferentes sobre las cajas denotan diferencias significativas ( $p<0,05)$.

relativamente cercanos, factiblemente, en respuesta a diferencias en la disponibilidad de alimento. En Torca y en La Floresta, se registraron divergencias en la actividad de los murciélagos entre puntos de observación, separados por menos de $60 \mathrm{~m}$. En Las Mercedes y en Torca, el movimiento de los murciélagos fue mayor, al menos a una hora de la noche, cerca a lugares con lámparas que en sitios sin ellas. Además, en estos áreas la actividad de forrajeo se concentró casi en su totalidad, alrededor de los puntos con lámparas. Adicionalmente, los lugares con lámparas: en Torca, en Las Mercedes y en la U.D.C.A lograron niveles de forrajeo mayores, a los registrados en los otros lugares. Así, los resultados también indican que los puntos de observación con iluminación artificial son usados como parches de forrajeo por parte de los murciélagos, como ocurre en otras áreas urbanas y semiurbanas del neotrópico (Avila-Flores $\mathcal{E}$ Fenton, 2005; Jung \& Kalko, 2010) y de la región holártica (López-García, 2006; Rydell, 2006).

Cómo ya se mencionó, las lámparas, en particular las de luz blanca, se convierten en parches alimentarios, valiosos para los murciélagos insectívoros, en ambientes urbanos o semiurbanos, dado que atraen y acumulan insectos a su alrededor y actúan mejor cuando están rodeadas de ambientes naturales o similares (Gaisler et al. 1998; Rydell, 2006). Así, las lámparas son aprovechadas en el norte de 
Bogotá por los murciélagos, como parches de alimentarios y parecen ser apreciables en sitios con baja afluencia vehicular y cerca a zonas de humedal, como en Las Mercedes y en la U.D.C.A. Esto sugiere que la preservación de áreas con remanentes naturales cerca a lámparas es importante para los murciélagos, posiblemente porque estas áreas son la fuente de sus insectos presa (Fenton, 1997).

En conclusión, como en trabajos realizados en áreas urbanas del neotrópico (Avila-Flores E Fenton, 2005) y de la región holártica (Gaisler et al. 1998), los murciélagos del borde norte de Bogotá reaccionan a la heterogeneidad antrópica del paisaje. Dichas modificaciones parecen tener efectos en dos direcciones: 1) las vías con alto tráfico vehicular pueden ser agentes de mortalidad y, por ello, podría reducir las posibilidades para la movilización de los murciélagos, durante algunas horas de la noche y, 2) la disposición de lámparas, en particular cerca a áreas de humedal, parece beneficiar, al menos, a algunos murciélagos, que encuentran allí una acumulación de presas potenciales. Estudios adicionales deben examinar si estas observaciones se cumplen para todas las especies de murciélagos de Bogotá, o si las respuestas de comportamiento dependen de la especie que se examine, como ocurre en otras áreas urbanas y semiurbanas (Duchamp et al. 2004; Jung \& Kalko, 2010).

Por último, los resultados de este trabajo sugieren que los murciélagos podrían ser aprovechados en las prácticas agriculturales de la región. Sitios con lámparas de luz blanca, además de concentrar insectos, pueden afectar la capacidad de algunas presas para reaccionar contra el ataque de los murciélagos. Algunas especies de polillas con "oídos", capaces de detectar llamadas de ultrasonido, tienen una menor probabilidad de evadir a los murciélagos cerca a una lámpara de luz blanca que lejos de ellas (Svensson $\mathcal{E}$ Rydell, 1998). Así, en un área con actividad agrícola, como el norte de Bogotá y donde los murciélagos parecen estar presentes en toda su extensión, la concurrencia de lámparas de luz blanca cerca a los cultivos podría ayudar a controlar las poblaciones de insectos nocturnos, como algunas polillas, que son consideradas plaga. La aplicación de este tipo de estrategia implicaría, potencialmente, una reducción en la necesidad de pesticidas, por lo tanto, un ahorro para los agricultores (Cleveland et al. 2006) y también menor contaminación de suelos y de aguas. Además, aportaría para reconocer los servicios ambientales que pueden ofrecer los murciélagos, lo que también puede ayudar a sensibilizar a los bogotanos sobre la importancia del capital natural que los rodea.

Agradecimientos: A los habitantes de los sitios, por su amabilidad y por permitir el acceso a los predios. A los integrantes del semillero de investigación Ecología y Conservación Ambiental-U.D.C.A (Sergio Barrantes, Geraldine Bobadilla, Maribel Casallas, Juanita Niño, María Fernanda Patiño, Lina Suárez y Lorena Téllez) y a Norma Gómez, quienes colaboraron en la toma de los datos. Dinesh Rao hizo sugerencias al "Summary" y al título. Dos evaluadores anónimos hicieron comentarios que ayudaron a mejorar el documento. Conflictos de intereses: No existe conflicto de intereses que ponga en riesgo la validez de los resultados presentados. Financiación: Este estudio deriva de los proyectos "Murciélagos del corredor ecológico del borde norte de Bogotá" y "Estudio del borde norte de Bogotá como un sistema complejo" financiados por la U.D.C.A y en parte por la CAR, en trabajo coordinado por el Instituto de Estudios Urbanos, Universidad Nacional de Colombia.

\section{BIBLIOGRAFÍA}

1. ADAMS, C.E.; LINDSEY, K.J.; ASH, S.J.. 2006. Urban wildlife management. CRC Press, Taylor $\mathcal{E}$ Francis Group (Boca Raton, FL). 311p.

2. ALCALDÍA MAYOR DE BOGOTÁ. 2000. Decreto 619 de 2000, Por el cual se adopta el Plan de Ordenamiento Territorial para Santa Fe de Bogotá, Distrito Capital. Disponible desde Internet en http:// www.alcaldiabogota.gov.co/sisjur/normas/Norma1. jsp?i=3769 (con acceso el 09/11/10).

3. ALTRINGHAM, J.D. 2001. Bats: biology and behaviour. Oxford University Press (New York): p.262.

4. ASOCIACIÓN BOGOTANA DE ORNITOLOGÍA ABO. 2000. Aves de la Sabana de Bogotá: guía de campo. Asociación Bogotana de Ornitología-Corporación Autónoma Regional de Cundinamarca CAR (Bogotá). 276p.

5. AVILA-FLORES, R.; FENTON, M.B. 2005. Use of spatial features by foraging insectivorous bats in a large urban landscape. J. Mammalogy. 86:1193-1204.

6. BROWN, J.H. 1968. Activity patterns of some Neotropical bats. J. Mammalogy. 49:754-757.

7. CLEVELAND, C.J.; BETKE, M.; FEDERICO, P.; FRANK, J.D.; HALLARA, T.G.; HORN, J.; LÓPEZ Jr., J.D.; MCCRACKEN, G.F.; MEDELLÍN, R.A.; MORENOVALDEZ, A.; SANSONE, C.G.; WESTBROOK, J.K.; KUNZ, T.H. 2006. Economic value of the pest control service provided by Brazilian free-tailed bats in southcentral Texas. Front. Ecol. Environ. 4:238-243. 
8. CORPORACIÓN AUTÓNOMA REGIONAL DE CUNDINAMARCA CAR. 2006. Los Cerros Orientales de Bogotá D.C.: patrimonio cultural y ambiental del Distrito Capital, la región y el país: plan de manejo ambiental, p.116. Alcaldía Mayor de Bogotá (Bogotá). $116 p$.

9. DUCHAMP, J.E.; SPARKS, D.W.; WHITAKER Jr., J.O. 2004. Foraging-habitat selection by bats at an urbanrural interface: comparison between a successful and less successful species. Can. J. Zool. 82:1157-1164.

10. ERKERT, H.G. 1978. Sunset-related timing of flight activity in Neotropical bats. Oecologia. 37:59-67.

11. ESTRADA, A.; JIMÉNEZ, C.; RIVERA, A.; FUENTES, E. 2004. General bat activity measured with an ultrasound detector in a fragmented tropical landscape in Los Tuxtlas, Mexico. Animal Biodiversity and Conservation. 27:1-9.

12. FENTON, M.B. 1974. Feeding ecology of insectivorous bats. Bios. 45:3-15.

13. FENTON, M.B. 1997. Science and the conservation of bats. J. Mammalogy. 78(1):1-14.

14. FENTON, M.B.; ACHARYA, L.; AUDET, D.; HICKEY, M.B.C.; MERRIMAN, C.; OBRIST, M.K.; SYME, D.M.; ADKINS. B. 1992. Phyllostomid bats (Chiroptera: Phyllostomidae) as indicators of habitat disruption in the Neotropics. Biotropica. 24:440-446.

15. FENTON, M.B.; BELL, G.P. 1981. Recognition of species of insectivorous bats by their echolocation calls. J. Mammalogy. 62:233-243.

16. FLEMING, T. 1993. Plant-visiting bats. Am. Scient. 81:460-467.

17. GAISLER, J.; ZUKAL, J.; REHAK, Z.; HOMOLKA, M. 1998. Habitat preference and flight activity of bats in a city. J. Zool. 244:439-445.

18. GARDNER, A.L. 1977. Feeding habits. En: Baker, R.J.; Jones, Jr. J.K.; C. Carter, D.C. (eds.). Biology of bats of the New World family Phyllostomatidae. Texas Tech University (Lubbock, Texas, USA): p.293-350.

19. JENNINGS, N.; POCOCK, M.J.O. 2009. Relationships between sensitivity to agricultural intensification and ecological traits of insectivorous mammals and arthropods. Conserv. Biol. 23:1195-1203.
20. JUNG, K.; KALKO, E.K.V. 2010. Where forest meets urbanization: foraging plasticity of aerial insectivorous bats in an anthropogenically altered environment. J. Mammalogy. 91:144-153.

21. KUNZ, T.H. 1973. Resource utilization: temporal and spatial components of bat activity in Central lowa. J. Mammalogy. 54:14-32.

22. LODÉ, T. 2000. Effect of a motorway on mortality and isolation of wildlife populations. Ambio. 29:163-166.

23. LÓPEZ-GARCÍA, C. 2006. Efectos de la iluminación viaria en los murciélagos. Boletín S.C.V. 11:38-43.

24. MEDELLÍN, R.A.; EQUIHUA, M.; AMIN, M.A. 2000. Bat diversity and abundance as indicators of disturbance in Neotropical rainforests. Conserv. Biol. 14:16661675.

25. MENDOSA, J.E.; ETTER, A. 2002. Multitemporal analysis (1940-1996) of land cover changes in the southwestern Bogotá highplain (Colombia). Landscape Urban Plan. 59:147-158.

26. NORBERG, U.M.; RAYNER, J.M.V. 1987. Ecological morphology and flight in bats (Mammalia: Chiroptera): wing adaptations, flight performance, foraging strategy and echolocation. Phil. Trans. R. Soc. Lond. B. 316:335-427.

27. OSBAHR, K.; HERNÁNDEZ SCHMIDT, M. 2006. Caracterización florística de un fragmento del humedal Torca-Guaymaral (Bogotá, Cundinamarca). Rev. U.D.C.A. Act. \& Div. Cient. 9:117-128.

28. PACHECO, V.; PATTERSON, B.D. 1992. Systematics and biogeographic analyses of four species of Sturnira (Chiroptera, Phyllostomidae), with emphasis on Peruvian forms. En: Young, K.R.; Valencia, N. (eds.). Biogeografía, ecología y conservación del bosque montano en el Perú. Universidad Nacional Mayor de San Marcos (Lima). p.57-81.

29. PULLIN, A.S. 2002. Conservation Biology. Cambridge University Press (Cambridge). p.345.

30. RAMÍREZ, D.P.; TRESPALACIOS, O.L.; RUIZ, F.L.; OTERO, J. 2008. Biodiversidad y conectividad ecológica en la zona urbano rural de la localidad de Suba. Instituto de Investigación de Recursos Biológicos Alexander von Humboldt (Bogotá D.C.). 154p. 
31. RUSSELL, A.L.; BUTCHKOSKI, C.M.; SAIDAK, L.; MCCRACKEN, G.F. 2009. Road-killed bats, highway design, and the commuting ecology of bats. Endangered Species Res. 8:49-60.

32. RYDELL, J. 2006. Bats and their insect prey at streetlights. En: Longcore, R.C. (ed). Ecological consequences of artificial night lighting. Island Press (Washington). p.43-60.

33. SECRETARÍA DISTRITAL DE AMBIENTE. 2007. Atlas ambiental de Bogotá D.C. Secretaría Distrital de Ambiente, Alcaldía Mayor de Bogotá D.C. (Bogotá D.C.). 272p.

34. SVENSSON, A.M.; RYDELL, J. 1998. Mercury vapour lamps interfere with the bat defence of tympanate moths (Operophtera spp.; Geometridae). Anim. Behav. 55:223-226.

35. TAMSITT, J.R.; VALDIVIESO, D. 1963. Records and observations on Colombian bats. J. Mammalogy. 44:168-180.
36. TAMSITT, J.R.; VALDIVIESO, D.; HERNÁNDEZCAMACHO, J.I. 1964. Bats of the Bogota savanna, Colombia, with notes on altitudinal distribution of Neotropical bats. Rev. Biol. Trop. 12:107-115.

37. TIME AND DATE AS. [En línea]. Sunrise and sunset in Bogota. Disponible desde Internet en http://www. timeanddate.com/worldclock/astronomy.html?n=41 (con acceso 15/07/10).

38. TUTTLE, M.D.; MORENO, A. 2007. Murciélagos cavernícolas del norte de México: su importancia y problemas de conservación. Bat Conservation International (Austin, Texas). 49p.

39. ZAR, J.H. 1999. Biostatistical analysis, 4th ed. Prentice Hall (Upper Saddle River, N. J.). 663p.

40. ZURCHER, A.; SPARKS, D.W.; BENNET, V.J. 2010. Why did the bat did not cross the road? Acta Chiropterologica 12:337-340.

Recibido: Octubre 15 de 2010

Aceptado: Enero 22 de 2011 